BULL. AUSTRAL. MATH. SOC.

VOL. $22(1980), 431-438$.

\title{
LEGENDRE POLYNOMIALS IN IRRATIONALITY PROOFS
}

\author{
F. BEUKERS
}

It is shown that a simple trick involving Legendre polynomials readily yields the irrationality of $e^{a}, a \in \mathbb{P}, \pi^{2}$, and of the zeros of Bessel functions of integer order. Generalisation of this idea yields the irrationality of $\zeta(3)$.

1.

In this paper we give simple proofs for the irrationality of $e^{a}$ $(a \in \mathbb{Q}), \pi^{2}$ and the zeros of Bessel functions of integer order. Of course these results are not new. Lamberti [7] showed the irrationality of $\pi$ and $e^{a}(a \neq 0, a \in 0)$ about two hundred years ago. Legendre [8] showed the irrationality of $\pi^{2}$ and the irrationality of the zeros of Bessel functions are implicit in his 'Note IV'. These classical proofs use continued fraction expansions of certain analytic functions. Other proofs were given for example by Niven [9] (for $\pi$ ) and Gerritzen [6] (for the zeros of Bessel functions). Also Siegel [10] produces some irrationality proofs (pp. 8, 9, 10) and in the same book one finds transcendence proofs for the numbers mentioned.

The proofs that we give here make use of a simple trick involving Legendre-polynomials. It is possible to generalize this method to other orthogonal polynomials with non-trivial weights. Also, one can obtain irrationality measures for certain numbers by this method, but for the sake

Received 19 June 1980. The author was supported in part by NSF grant MCS77-18723(02). 
of simplicity we do not exploit these possibilities. For irrationality measures one might look at Alladi and Robinson [1], Bundschuh [3] or Choodnovski [4]. Finally, it is possible to give two- and threedimensional generalisations of our method to produce irrationality proofs for $\zeta(2)$ and $\zeta(3)$; see Beukers [2].

2.

The Legendre-polynomial for the interval $[0,1]$ is defined by

$$
P_{n}(x)=(1 / n !)(d / d x)^{n} x^{n}(1-x)^{n}
$$

After working out the differentiations we find

$$
P_{n}(x)=\sum_{m=0}^{n}\left(\begin{array}{c}
n \\
m
\end{array}\right)\left(\begin{array}{c}
n+m \\
n
\end{array}\right)(-x)^{m} .
$$

Notice that the degree of $P_{n}(x)$ is $n$ and that $P_{n}(x) \in \mathbb{Z}[x]$. These are the only things we need in our computations. For more facts about Legendre-polynomials and other orthogonal polynomials see, for example, [5] or $[11]$.

THEOREM 1. Let $a \in \mathbb{Q}, a \neq 0$. Then $e^{a} \notin \mathbb{Q}$.

Proof. It is sufficient to prove our theorem for $a \in \mathbb{N}$. Consider

$$
I_{n}=\int_{0}^{1} e^{a t_{P_{n}}(t) d t}
$$

Insert the expression (1) for $P_{n}(t)$ and perform a repeated partial integration until we obtain

$$
I_{n}=(-1)^{n}\left(a^{n} / n !\right) \int_{0}^{1} e^{a t} t^{n}(1-t)^{n} d t
$$

Since the integrand is positive, we have $I_{n} \neq 0$. The following estimate is obvious;

$$
\left|I_{n}\right|<|a / n|^{n}\left(e^{a} / n !\right)
$$

On the other hand $I_{n}$ consists of terms of the type 


$$
\int_{0}^{1} e^{a t} t^{m} d t=1 / a^{m+1} \int_{0}^{a} e^{t} t^{m} d t \quad(m \leq n) .
$$

By partial integration it is easy to see that

$$
\int_{0}^{a} e^{t} t^{m} d t=C+D e^{a}
$$

where $C, D \in \mathbb{Z}$. Hence

$$
I_{n}=\left(1 / a^{n+1}\right)\left(R_{n}+S_{n} e^{a}\right)
$$

where $R_{n}, S_{n} \in \mathbb{Z}$. Now suppose $e^{q}=p / q$. Since $I_{n}$ is nonzero it can be estimated below by

$$
1 / q a^{n+1} \leq\left(1 / a^{n+1}\right)\left|R_{n}+S_{n}(p / q)\right|=\left|I_{n}\right|,
$$

which contradicts the upper bound (2) for $I_{n}$ if $n$ is sufficiently

large. Hence $e^{a}$ is irrational.

THEOREM 2. $\pi^{2}$ is irrational.

Proof. Consider

$$
I_{n}=\int_{0}^{1}(\sin \pi t) P_{2 n}(t) d t
$$

By substituting the expression (1) for $P_{n}$ and after $2 n$-fold partial integration we obtain

$$
I_{n}=(-1)^{n}\left(\pi^{2 n} /(2 n) !\right) \int_{0}^{1}(\sin \pi t) t^{2 n}(1-t)^{2 n} d t
$$

and hence the estimate

$$
\left|I_{n}\right|<(\pi / 4)^{2 n}(1 /(2 n) !)
$$

Also note that $I_{n} \neq 0$. On the other hand $I_{n}$ consists of terms of the shape 


$$
\int_{0}^{l} t^{m}(\sin \pi t) d t, m \leq 2 n \text {. }
$$

By partial integration we see that

$$
\int_{0}^{1} t^{m}(\sin \pi t) d t=(1 / \pi)-\left(m(m-1) / \pi^{2}\right) \int_{0}^{1} t^{m-2}(\sin \pi t) d t .
$$

Hence $I_{n}=\pi^{-1} A_{n}\left(\pi^{-2}\right)$, where $A_{n}$ is an $(n-1)$ st degree polynomial with integral coefficients. Now suppose $\pi^{2}=p / q$. Since $I_{n}$ is nonzero it can be estimated below by

$$
1 / \pi p^{n} \leq\left|(I / \pi) A_{n}(q / p)\right|=\left|I_{n}\right|
$$

contradicting the upper bound for $I_{n}$ if $n$ is sufficiently large. Hence $\pi^{2}$ is rational.

The following theorem deals essentially with the Bessel-function

$$
J_{r}(z)=\sum_{k=0}^{\infty}(-1)^{n}(z / 2)^{r+2 n} / n !(n+r) !
$$

where $r=0,1,2, \ldots$. In order to make computations simpler however, we shall state the theorem in terms of the simplified function

$$
L_{r}(z)=\sum_{n=0}^{\infty} z^{n} / n !(n+r) ! .
$$

The connection between $J_{p}(z)$ and $L_{p}(z)$ is given by $L_{r}\left(-z^{2} / 2\right)=z^{-r} J_{r}(z)$. Also, we shall make use of the modified polynomial

$$
P_{n, r}(x)=\left(x^{-r} / n !\right)(d / d x) x^{r+n}(1-x)^{n} .
$$

It is easy to see that $P_{n, r}(x)$ has degree $n$ and integral coefficients.

THEOREM 3. The zeros of $L_{\gamma}(z)$ are irrational.

Proof. Consider 


$$
I_{n}(z)=z^{n} \int_{0}^{1} t^{r} L_{r}(z t) P_{n, r}(t) d t
$$

Substitute (.3) and perform an n-fold partial integration. Then

$$
I_{n}(z)=z^{2 n}\left((-1)^{n} / n !\right) \int_{0}^{1} D^{n} L_{r}(z t) t^{n+r}(1-t)^{n} d t
$$

where $D$ denotes differentiation. From the Taylor expansion of $L_{p^{p}}(z)$ it is easy to see that

$$
\left|D^{n} L_{p}(z)\right| \leq e^{|z|}
$$

for any $z \in \mathbb{C}$ and any $n \geq 0$. Hence

$$
\left|I_{n}\right|<|z / 2|^{2 n}(2 / n !) e^{|z|} .
$$

On the other hand $I_{n}(z)$ consists of terms of the shape

$$
z^{n} \int_{0}^{1} L_{r}(z t) t^{m+r} d t=z^{n-m-r-1} \int_{0}^{z} L_{r}(t) t^{m+r} d t, m \leq n .
$$

By using the differential equation $z^{-r} D\left(z^{r+1} D L_{r}\right)=L_{r}$ and partial integration, we see that

$$
\int_{0}^{z} L_{r}(t) t^{m+r} d t=z^{m+r+1} L_{r}^{\prime}(z)-m z^{m+r} L_{r}(z)+m(m+r) \int_{0}^{z} t^{m+r-1} L_{r}(t) d t
$$

and hence

$$
\int_{0}^{z} L_{r}(t) t^{m+r} d t=U_{m}(z) z^{p+1} L_{r}^{\prime}(z)+V_{m}(z) z^{p+1} L_{r}(z)
$$

where $U_{m}(z), V_{m}(z)$ are polynomials of degree $m, m-1$ respectively with integral coefficients. Notice also that $V_{m}(0) \neq 0$. Collecting terms we find that

$$
I_{n}(z)=A_{n}(z) L_{p}^{\prime}(z)+B_{n}(z) L_{p}(z)
$$

where $A_{n}(z), B_{n}(z)$ are of degree $n, n-1$ respectively and have integral coefficients. Notice that $B_{n}(0) \neq 0$ for all $n$. 
Now suppose $\alpha$ is a rational zero of $L_{r}(z)$. It is clear that $L_{r}^{\prime}(\alpha) \neq 0$ because otherwise the differential equation would imply $L_{p}(z) \equiv 0$. Inequality (5) now implies

$$
\left|A_{n}(\alpha) L_{r}^{\prime}(\alpha)\right|<|\alpha / 2|^{2 n+1}(2 / n !) e^{|\alpha|}
$$

Since $\alpha$ is rational we have either $A_{n}(\alpha)=0$ or $\left|A_{n}(\alpha)\right| \geq b^{-n}$, where $b$ is the denominator of $\alpha$. For sufficiently large $n$ however, the second possibility contradicts the upper bound we have for $A_{n}(\alpha)$. Therefore $A_{n}(\alpha)=0$ for all sufficiently large $n$, and so is $I_{n}(\alpha)$. We will show that this is not possible, and thus prove our theorem.

Consider (6) for $n$ and $n-1$, and eliminate $L_{p}(z)$. We obtain

$$
D_{n}(z) L_{r}^{\prime}(z)=B_{n-1}(z) I_{n}(z)-B_{n}(z) I_{n-1}(z) \text {, }
$$

where we have put $D_{n}(z)=A_{n}(z) B_{n-1}(z)-A_{n-1}(z) B_{n}(z)$. From (4) we see that $D_{n}(z) L_{r}^{\prime}(z)$ is divisible by $z^{2 n-2}$, and since $L_{r}^{\prime}(0) \neq 0$, the polynomial $D_{n}(z)$ is divisible by $z^{2 n-1}$. Since $D_{n}(z)$ has degree at most $2 n-2$, we infer $D_{n}(z)=c_{n} z^{2 n-2}$ for some constant $c_{n}$. Substitute this in (7), divide by $z^{2 n-2}$ and put $z=0$. Then we end up with

$$
\begin{aligned}
c_{n} L_{r}^{\prime}(0) & =-B_{n}(0)\left((-1)^{n-1} /(n-1) !\right) \int_{0}^{1} D^{n-1} L_{r}(0) t^{n+r}(1-t)^{n} d t \\
& =-B_{n}(0)\left((-1)^{n-1} /(n-1) !\right) D^{n-1} L_{r}(0)((n+r) ! n ! /(2 n+r+1) !) .
\end{aligned}
$$

Since all factors on the right-hand side are nonzero we conclude that $c_{n} \neq 0$ for all $n$. Hence $D_{n}(\alpha) \neq 0$, and from expression (7) with $z=\alpha$ we see that at least one of $I_{n}(\alpha), I_{n-1}(\alpha)$ is not zero, which concludes the proof of our theorem. 


\section{References}

[1] K. Alladi and M.L. Robinson, "On certain irrational values of the logarithm", Number theory, Carbondale 1979, 1-9 (Proc. Southern Illinois Number Theory Conference, Carbondale, 1979. Lecture Notes in Mathematics, 751. Springer-Verlag, Berlin, Heidelberg, New York, 1979).

[2] F. Beukers, "A note on the irrationality of $\zeta(2)$ and $\zeta(3)$ ", Bulz. London Math. Soc. 11 (1979), 268-272.

[3] Peter Bundschuh, "Irrationalitätsmaße für $e^{a}, a \neq 0$ rational oder Liouville-Zahl", Math. Ann. 192 (1971), 229-242.

[4] Gregory V. Choodnovsky, "Approximations rationnelles des logarithmes de nombres rationnels", C.R. Acad. Sci. Paris Ser. A 288 (1979), $607-609$.

[5] Arthur Erdelyi, Wilhelm Magnus, Fritz Oberhettinger, Francesco G. Tricomi, Higher Transcendental Functions, Volume II (McGrawHill, New York, Toronto, London, 1953).

[6] L. Gerritzen, "Ein p-adischer Beweis für die Irrationalität der Nullstellen von Besselfunktionen", Math. Ann. 266 (1977), 253-255.

[1] lohannis Henrici Lamberti, "Mémoire sur quelques propriétés remarquables des quantités transcendantes circulaires et logarithmiques", Opera Mathematica, Volumen Secundum, 112-159 (Orell Füßli Verlag, Zürich; 1948).

[8] A.M. Legendre, "Les polygones réguliers et la mesure du cercle", Élements de géométrie, Livre IV (Librairie de Firmin Didot Frères, Paris, 1850).

[9] I van Niven, "A simple proof that $\pi$ is irrational", Buzz. Amer. Math. Soc. 53 (1947), 509.

[10] Carl Ludwig Siegel, Transcendental numbers (Annals of Mathematics Studies, 16. Princeton University Press, Princeton, 1949). 
[11] Gabor Szegö, Orthogonal polynomials (Amer. Math. Soc. Colloquium Publications, 23. American Mathematical Society, New York, 1939. Reprinted, 1959).

Mathematisc Institut, Rijksuniversiteit Leiden, Wassenarseweg 80, Postbus 9512, 2300 RA Leiden, Netherlands. 